\title{
MiR-421 Is Overexpressed and Promotes Cell Proliferation in Non-Small Cell Lung Cancer
}

\author{
Xing Li Shao-Hua Chen Jin-Wu Zeng \\ Department of Respiratory Medicine, Jingzhou Central Hospital, The Second Clinical Medical College, \\ Yangtze University, Jingzhou, PR China
}

\section{Highlights of the Study}

- The present study showed that miR-421 was overexpressed in NSCLC.

- Upregulated miR-421 was associated with a shorter overall survival time of patients with NSCLC.

- Bioinformatics analysis revealed miR-421 was involved in transcription, cell cycle, and insulin signaling pathway regulation.

- A gain of function assay showed that miR-421 could promote NSCLC cell proliferation and cell cycle progression.

\section{Keywords}

Lung cancer $\cdot$ MiR-421 $\cdot$ Proliferation $\cdot$ Cell cycle $\cdot$ Biomarker

\section{Abstract}

Background: Lung cancer is the main cause of cancerrelated deaths worldwide, and the overall 5-year survival rate of non-small cell lung cancer (NSCLC) remained low. MicroRNAs had been confirmed to be an important regulator in tumor progression, and they could serve as either tumor promoters or suppressors in NSCLC. Objectives: To identify the novel cancer-specific biomarkers for NSCLC patients, which may be useful to monitor tumor progression and improve NSCLC patients' survival. Method: The expression profile of miR-421 was analyzed in NSCLC samples using public datasets, including The Cancer Genome Atlas and GSE102286. The expression level of miR-421 was detected by reverse transcription-polymerase chain reaction. Cell proliferation and cell cycle were detected by Cell Counting Kit assay, flow cytometry assay, respectively. Kyoto Encyclopedia

\section{KARGER}

E-Mail karger@karger.com www.karger.com/mpp
(C) 2019 The Author(s) Published by S. Karger AG, Basel

Karger Open access

This is an Open Access article licensed under the Creative Commons Attribution-NonCommercial-4.0 International License (CC BY-NC) (http://www.karger.com/Services/OpenAccessLicense), applicable to the online version of the article only. Usage and distribution for commercial purposes requires written permission. of Genes and Genomes analysis were applied to determine the biological roles of miR-421, based on the online DAVID system. Statistical comparisons between groups of normalized data were performed using $t$ test or Mann-Whitney $U$ test according to the test condition. Results: In this study, we focused on exploring the roles of miR-421 in NSCLC prognosis and growth. The present study for the first time showed that miR-421 was overexpressed in NSCLC and associated with a shorter overall survival time of patients with NSCLC. Bioinformatics analysis revealed miR-421 was involved in transcription, cell cycle, and insulin signaling pathway regulation. Furthermore, a gain of function assay showed that overexpression of miR-421 could promote NSCLC cell proliferation and cell cycle progression. Conclusions: Our findings suggest that miR-421 might be a promising prognostic and therapeutic target for NSCLC.

(C) 2019 The Author(s)

Published by S. Karger AG, Basel

Xing Li and Shao-Hua Chen are co-first authors.

Department of Respiratory Medicine, Jingzhou Central Hospital

The Second Clinical Medical College, Yangtze University

Jingzhou, Hubei 434020 (PR China)

E-Mail lixinglijing@126.com 


\section{Introduction}

Lung cancer has become the main cause of cancer-related deaths worldwide. In the United States, lung cancer is estimated to account for $26 \%$ of cancer-related deaths [1]. In China, the mortality of lung cancer has increased by $>4$ times during the past 3 decades [2]. Non-small cell lung cancer (NSCLC), including lung squamous cell carcinoma (LUSC), lung adenocarcinoma (LUAD), and large cell lung cancer, is the main type of lung cancer and constitutes $80 \%$ of lung cancer cases [3]. Despite a series of new diagnostic and treatment strategies developed in anticancer therapy over the past decades, the overall 5 -year survival rate of NSCLC remained as low as about $20 \%$ [1]. Thus, there is an urgent need to identify novel cancer-specific biomarkers for NSCLC patients, which may be useful to monitor tumor progression and improve NSCLC patients' survival.

MicroRNAs (miRNAs/miRs) are small noncoding single-stranded RNA molecules with 18-25 nucleotides in length. Emerging studies have highlighted the important roles of miRNAs in the tumorigenesis and progression of cancer. Multiple miRNAs have been reported to be involved in lung cancer progression. Certain miRNAs could serve as either tumor promoters or suppressors in NSCLC. For example, Zhang et al. [4] found the tumorinitiating cell-specific miR-1,246 and miR-1,290 acted as crucial drivers for tumor initiation and cancer progression in human NSCLC. MiR-30a suppressed lung cancer progression by targeting SIRT1 [5]. MiR-21 inhibitor restrains cell growth and invasion in NSCLC cells [6]. Exploring the functions of miRNAs in NSCLC could provide useful information to identify novel therapeutic targets for this disease.

Recent studies revealed the important roles of miR421 in cancer biology. MiR-421 could regulate apoptosis, autophagy, proliferation, metastasis, epithelial - mesenchymal transition, metabolism, and radiotherapy resistance in various types of cancer by targeting different genes, such as TLR [7], caspase-10 [8], caspase-3 [9], MEF2D [10], PFKFB2 [11], and FOXO4 [12]. miR-421 was observed to be abnormally expressed in human cancers, including neuroblastomas, breast cancer, liver cancer, and pancreatic cancer. For example, the serum expression level of miR-421 was significantly higher in osteosarcoma patients than those in healthy volunteers [13]. Jiang et al. [14] found that miR-421 was upregulated in human gastric carcinoma than in normal samples [9]. However, the expression pattern and functional roles of miR-421 in NSCLC remain unclear.
In the present study, we analyzed miR-421 expression pattern in NSCLC samples by analyzing public datasets. Furthermore, we performed a bioinformatic analysis to reveal the potential mechanisms of miR-421 in NSCLC. Finally, we conducted experiments to explore the effects of miR-421 on NSCLC cell cycle and proliferation. We thought this study would provide useful information to explore whether miR-421 could serve as a biomarker for NSCLC.

\section{Material and Methods}

MiRNA and mRNA Profile DATA Collection

The expression data of miR-421 in NSCLC samples were downloaded from The Cancer Genome Atlas (TCGA, https://tcga-data. nci.nih.gov/tcga/) database. A series of patient clinical features, including age at diagnosis, pathological tumor (T) stage, pathological node $(\mathrm{N})$ stage, and days to the last follow-up, were retrospectively obtained from patient records. All the patients were staged using the 2009 Tumor-Node-Metastasis classification of the American Joint Committee on Cancer/International Union against Cancer.

\section{Cell Culture}

A549 and H1299 cell lines were obtained from the American Type Culture Collection. A549 and NCI-H1299 cells were cultured in RPMI 1,640 medium supplemented with 10\% FBS ExCell Bio (ExCell, China) in a $37^{\circ} \mathrm{C}$ incubator with $5 \% \mathrm{CO}_{2}$.

\section{Cell Transfection}

Synthetic miR-421 mimic (5'-AUCAACAGACAUUAAUUGGGCGC- ${ }^{\prime}$; $\quad 5^{\prime}$-GCCCAAUUAAUGUCUGUUGAUUU- ${ }^{\prime}$ ) and miR-NC ( $5^{\prime}$-UUCUCCGAACGUGUCACGUTT- $\left.3^{\prime}\right)$ were purchased from MajorBio. miR-421 inhibitor (si-miR-421) and negative control for si-miR-421 (siNC) were synthesized by Sangon Biotech Co., Ltd. (Shanghai, China). Cells were seeded at 3 * $10^{5}$ cells/wells in 6-well plates and incubated overnight. Cells were transfected with siRNAs using HG-TransGene transfection reagent (Genomeditech). Forty-eight hours later, RNA was extracted to detect the transfection efficiency.

\section{RNA Extraction and Quantitative Reverse}

Transcription-Polymerase Chain Reaction

Total RNA for reverse transcription (RT)-quantitative polymerase chain reaction (qPCR) was extracted using TRIzol (Invitrogen) according to the manufacturer's instructions. RT was performed with PrimeScript reagent kit (TAKARA, Japan) following the manufacturer's instructions. miR-421-specific RT primer was 5'-GTCTCCTCTGGTGGAGGGTCCGAGGTATTCCCACCAGAGGAGACGCGCCC-3'. For analysis of microRNA expression, RT-qPCR was performed using AceQ qPCR SYBR Green Master Mix (Vazyme, China) on the LightCyclerR 480 (Roche, Switzerland). The expression level of $\mathrm{miR}-421$ was normalized to U6. The PCR primers for mature miR-421 (5'-GCGCGGATCAACAGACATTAATT- $3^{\prime}$; $5^{\prime}$-GTGCAGGGTCCGAGGT-3') and normalizer U6 (5'-CGCTTCGGCAGCACATATACTAA- $3^{\prime} ; 5^{\prime}$ TATGGAACGCTTCACGAATTTGC- $3^{\prime}$ ) were designed and pur- 


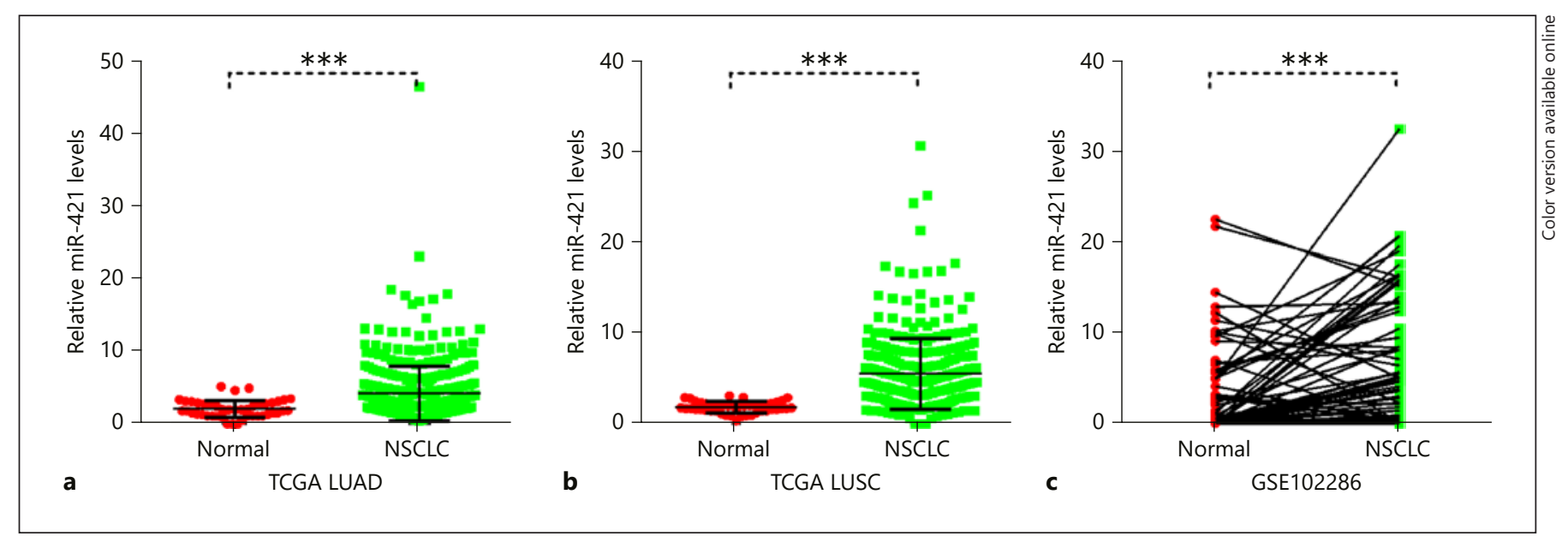

Fig. 1. miR-421 was upregulated in NSCLC. miR-421 was upregulated in tumor tissues compared with nontumor tissues in TCGA LUAD (a), LUSC dataset (b) and GSE102286 (c). ${ }^{* * *} p<0.001$. NSCLC, non-small cell lung cancer; LUAD, lung adenocarcinoma; TCGA, The Cancer Genome Atlas.

chased from HuaGene (HuaGene, China). Relative miRNA expression was calculated using the $2^{-\Delta \Delta \mathrm{Ct}}$ method [15]. Each sample was assayed in triplicate to ensure quantitative accuracy.

Cell Proliferation Assay

Six thousand transfected cells were seeded in 96-well plates at a final volume of $100 \mu \mathrm{L}$ medium/well. Proliferation rate was assessed at $0,24,48,72$, and 96 . Cell viability was quantified by adding $10 \mu \mathrm{L}$ Cell Counting Kit (Dojindo, Japan) according to the manufacturer's protocol. After a 1.5-h incubation, the plates were monitored at specific time points using a PowerWave XS Microplate reader (BioTek, Winooski, VT, USA), which measured absorbance at $450 \mathrm{~nm}$. The absorbance at $630 \mathrm{~nm}$ was used as a reference. Each experiment was performed at least in triplicate.

\section{Cell Cycle Assay}

Transfected A549 and H1299 cells in the log phase of growth were collected and fixed in $0.03 \%$ Triton X-100 and propidium iodide $(50 \mathrm{ng} / \mathrm{mL})$ for $15 \mathrm{~min}$. For the cell cycle analysis, the transfected cells were examined with a fluorescence-activated cell sorting flow cytometer (BD Biosciences, San Jose, CA, USA) and analyzed with ModFit software (Verity Software House, Topsham, ME, USA). Each test was performed in triplicate.

\section{Bioinformatics Analysis}

Gene ontology and Kyoto Encyclopedia of Genes and Genomes analyses were applied to determine the biological roles of miR-421, based on the online DAVID 6.8 online suit [16] (https://david.ncifcrf.gov/). The $p$ value (Hypergeometric- $p$ value) denoted the significance of the pathway correlated to the conditions. The recommend $p$ value cutoff is 0.05 .

\section{Statistical Analysis}

The numerical data of cell proliferation and cell cycle were presented as the mean \pm SD. All experiments are repeated at least 3 times. Statistical comparisons between groups of normalized data were performed using $t$ test or Mann-Whitney $\mathrm{U}$ test according to the test condition at $5 \%$ level of significance (i.e., $p<0.05$ ).

\section{Results}

\section{MiR-421 was Upregulated in NSCLC}

In this study, we downloaded TCGA LUAD and LUSC data to evaluated the expression pattern of miR-421 in NSCLC. Our results showed that miR-421 was upregulated in LUAD and LUSC tissues compared with nontumor tissues by analyzing TCGA datasets (Fig. 1a, b). Next, we analyzed a GEO dataset, GSE102286, which included 88 paired lung cancer samples. We observed a significant increase of miR-421 expression in NSCLC compared to normal tissues (Fig. 1c).

Furthermore, we evaluated the association of miR-421 expression levels with clinical features. As shown in Figure 2, we found $\mathrm{miR}-421$ expression level was overexpressed in patients with $\mathrm{N} 1+\mathrm{N} 2+\mathrm{N} 3+\mathrm{Nx}(p<0.05)$ NSCLC compared with $\mathrm{N}$ stage in both LUAD (Fig. 2a) and LUSC (Fig. 2b). We also found that miR-421 expression levels were overexpressed in patients with $\mathrm{T} 2+\mathrm{T} 3+$ T4 $(p<0.05)$ NSCLC compared with T1 NSCLC in both LUAD (Fig. 2d) and LUSC (Fig. 2e). Moreover, we observed miR-421 was upregulated in high-grade samples compared to low-grade samples (Stage I and II) in both LUAD (Fig. 2c) and LUSC (Fig. 2f).
Li/Chen/Zeng 


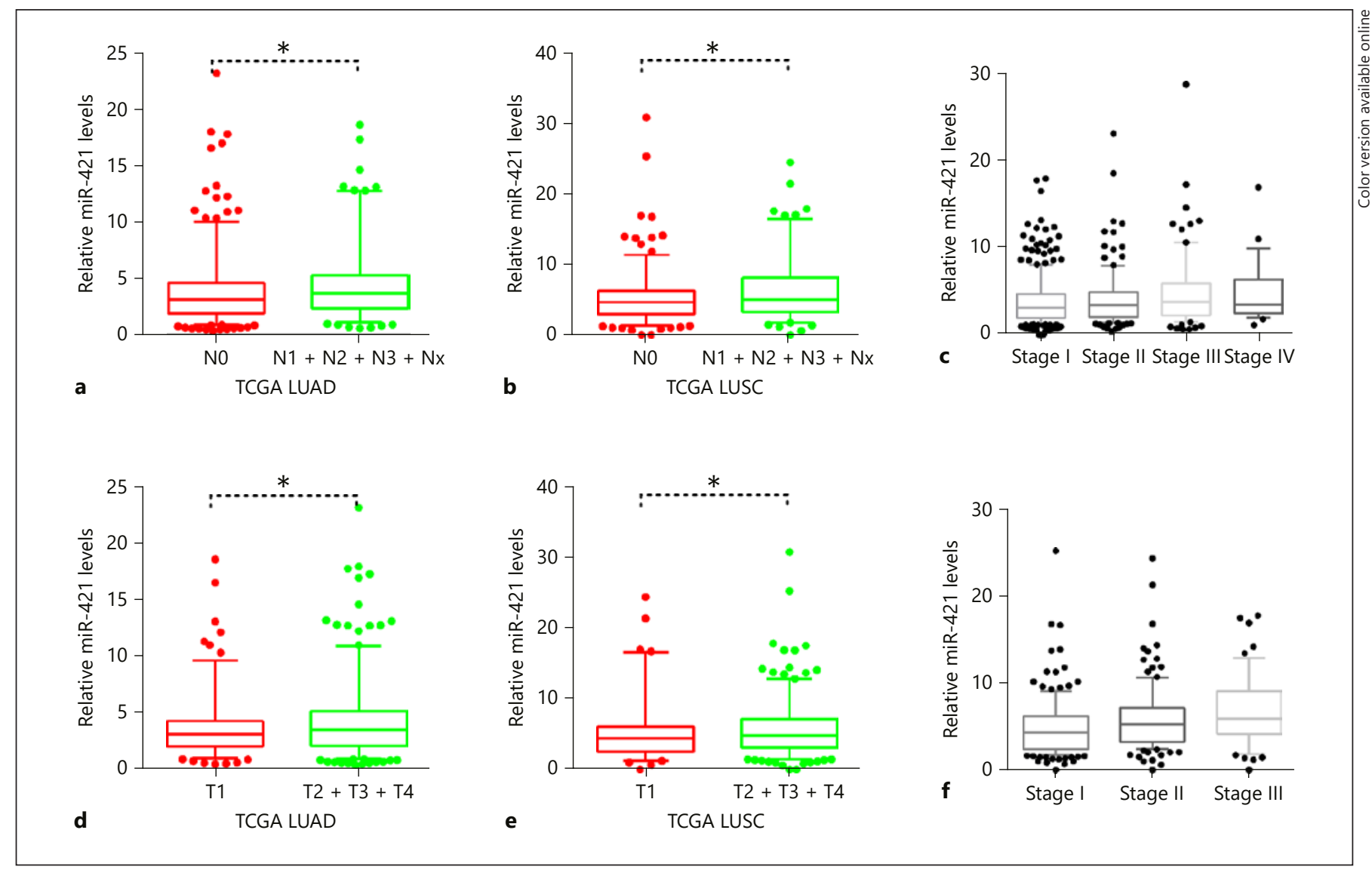

Fig. 2. miR-421 was upregulated in high tumor-node-metastasis stage NSCLC. a-c miR-421 expression levels were overexpressed in patients with $\mathrm{N} 1+\mathrm{N} 2+\mathrm{N} 3+\mathrm{Nx}$ compared to N0 NSCLC (a), in patients with $\mathrm{T} 2+\mathrm{T} 3+\mathrm{T} 4$ NSCLC compared with T1 NSCLC samples (b), and in high-grade samples compared to low-grade samples (c) by analyzing TCGA LUAD dataset. d-f miR-421 ex-

Kaplan-Meier method was performed to further investigate the prognostic value of miR-421 in NSCLC. As demonstrated in Figure 3, compared with patients with higher miR-421 expression, the overall survival and disease-free survival rates were higher in patients with lower miR-421 expression in TCGA LUAD and LUSC dataset. These results suggested the prognostic roles of miR-421 in NSCLC.

\section{Molecular Function Analysis of miR-421}

Furthermore, we performed a bioinformatics analysis for miR-421 to explore its potential mechanisms in NSCLC by using its targets. Two miRNA targets prediction websites were used, including TargetScan [17] and starbase [18] (Fig. 4b). A total of 260 targets of miR-421 were used to perform the gene ontologies categories analysis. pression levels were overexpressed in patients with $\mathrm{N} 1+\mathrm{N} 2+\mathrm{N} 3$ + Nx compared to N0 NSCLC $(\mathbf{d})$, in patients with T2 + T3 + T4 NSCLC compared with T1 NSCLC samples (e), and in high-grade samples compared to low-grade samples (f) by analyzing TCGA LUAD dataset. ${ }^{*} p<0.05$. LUSC, lung squamous cell carcinoma; LUAD, lung adenocarcinoma; TCGA, The Cancer Genome Atlas.

According to our analysis, we found that miR-421 was associated with transcription, protein mono-ubiquitination, positive regulation of translational initiation, liver development, positive regulation of G1/S transition of mitotic cell cycle, and negative regulation of translation (Fig. 4a). Molecular function assay showed miR-421 was involved in chromatin binding, poly(A) RNA binding, DNA binding, ubiquitin protein ligase binding, transcription factor activity, and nucleotide binding (Fig. 4c). Kyoto Encyclopedia of Genes and Genomes pathway analysis suggested miR-421 was mainly enriched in longterm potentiation, insulin signaling pathway, ubiquitinmediated proteolysis, oocyte meiosis, oxytocin signaling pathway, neurotrophin signaling pathway, signaling pathways regulating pluripotency of stem cells, glucagon signaling pathway, proteoglycans in cancer, and mito- 
gen-activated protein kinase (MAPK) signaling pathway (Fig. 4e). The targets of miR-421 involved in regulating insulin signaling pathway and the MAPK signaling pathway were shown in Figure $4 \mathrm{~d}$ and $\mathrm{f}$.

\section{Overexpression of miR-421 Promoted NSCLC Cell \\ Proliferation}

Aiming to evaluate the functions of miR-421 in NSCLC, we transfected A549 and H1299 cells with miR421 mimics. We observed a significant increase of miR421 gene expression in the transfected cells (Fig. 5a-b). To explore the potential effects of miR-421 on the proliferation of NSCLC, we performed the Cell Counting Kit assay and observed overexpression of miR-421 significantly promoted the proliferation of A549 (Fig. 5e) and H1299 cells (Fig. 5f). Moreover, we knockdown the expression of miR-421 in NSCLC cells and found that inhibition of miR-421 suppressed proliferation of A549 (Fig. 5c, g) and H1299 cells (Fig. 5d, h).

We then assessed the function of miR-421 on cell cycle profile of H1299 cells. Flow cytometric analysis revealed that overexpression of miR-421-3p in H1299 cells significantly promoted cell cycle progression by decreasing the proportion of cells in G1 phase and increasing the proportion of cells in S phase (Fig. 5i). Taken together, these results suggested miR-421 promoted NSCLC proliferation by inducing G1-phase cell cycle arrest.

\section{Overexpression of miR-421 Suppressed Insulin and}

MAPK-Signaling Regulators

In order to explore the mechanisms of miR-421 underlying NSCLC progression, we detected the effect of miR421 on insulin and MAPK-signaling regulators, including HK1, RAPGEF2, TRAF6, and RPS6KA3. As shown in Figure 6, we observed overexpression of miR-421 significantly suppressed the expression of HK1, RAPGEF2, TRAF6, and RPS6KA3 in A549 and H1299 cells. Moreover, we evaluated the association among miR-421 and these regulators in NSCLC samples. Our analyses showed that miR-421 expression was negatively correlated to the expression of HK1, RAPGEF2, TRAF6, and RPS6KA3 in NSCLC samples using TCGA dataset.

\section{Discussion}

In this study, we investigated the expression pattern of miR-421 in NSCLC. We found that miR-421 was significantly upregulated in lung cancer by analyzing TCGA and GEO datasets. Overexpression of miR-421 promoted

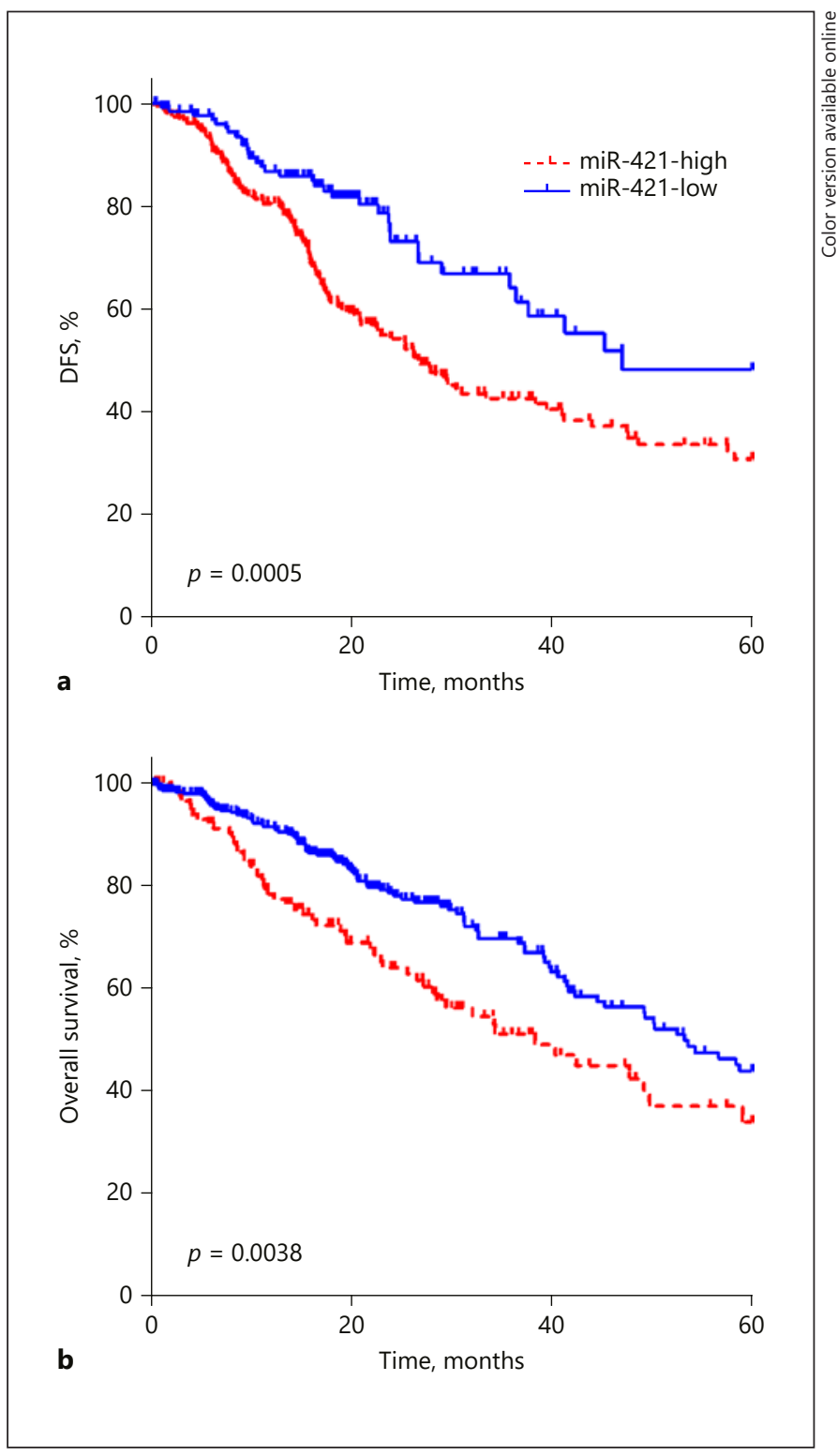

Fig. 3. Higher miR-421 expression was associated with poorer prognosis. The disease-free survival (a) and overall survival rates (b) were higher in patients with low miR-421 expression in this dataset compared with patients with high miR-421 expression. DFS, disease-free survival.

NSCLC proliferation. Bioinformatics analysis revealed that miR-421 was involved in regulating transcription, protein mono-ubiquitination, and cell cycle in NSCLC. This study was consistent with previous studies and extended the previous works. These results suggest that miR-421 might be a potential therapeutic target for NSCLC. 


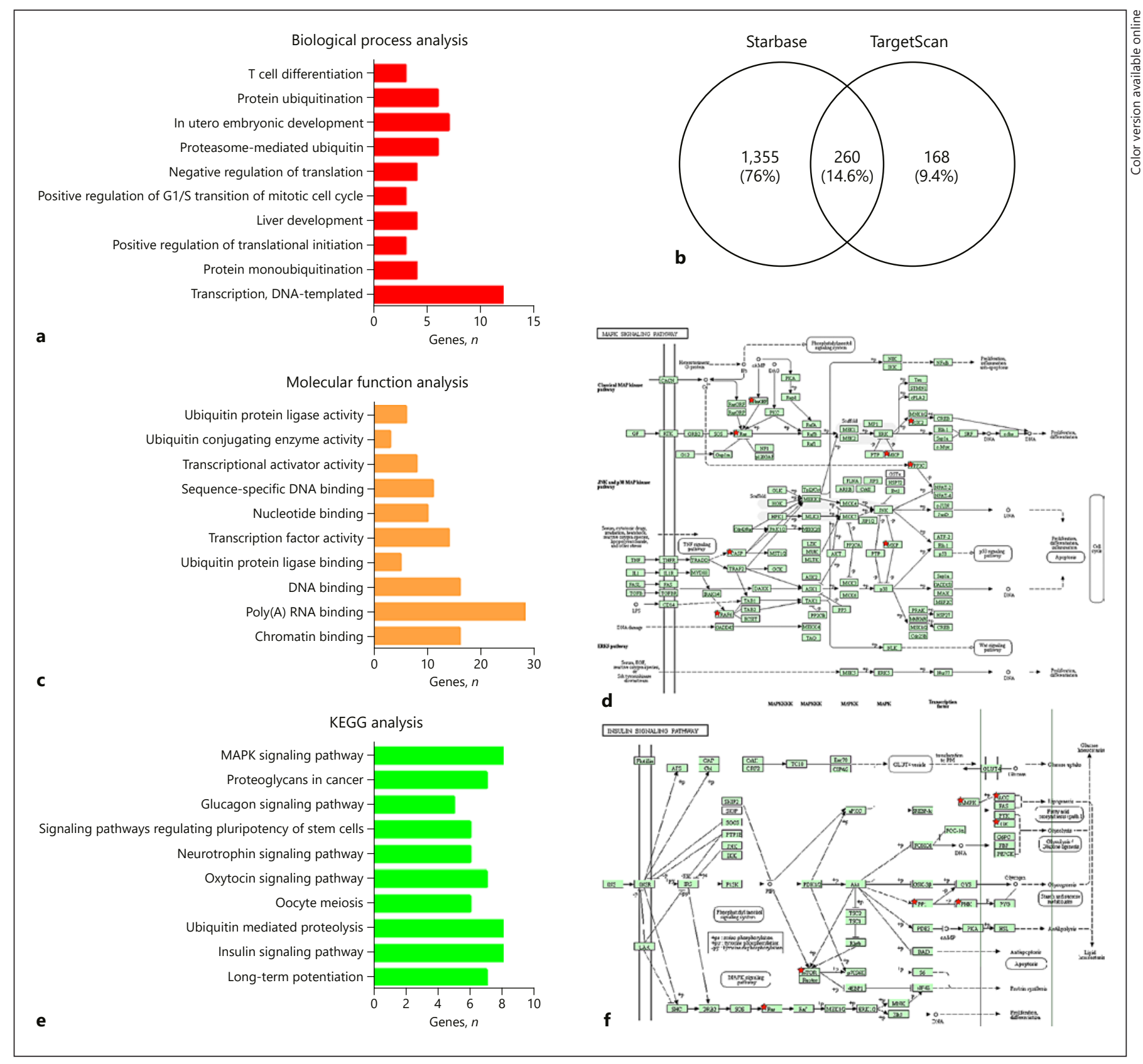

Fig. 4. Molecular function analysis of miR-421. a Gene ontologies categories analysis. b TargetScan and starbase were used to predict miRNA targets. c Molecular function analysis. e Kyoto Encyclopedia of Genes and Genomes analysis. The function of miR-421 in MAPK signaling pathway (d) and insulin signaling pathway (f). KEGG, Kyoto Encyclopedia of Genes and Genomes; MAPK, mitogen-activated protein kinase.

Recent studies have demonstrated the important roles of miRNAs in the development and progression of NSCLC. For example, miR-99a enhanced the radiation sensitivity of NSCLC by targeting mTOR [19], and microRNA-485-5p suppressed growth and metastasis in
NSCLC cells by targeting IGF2BP2 [20]. In the present study, we found that miR-421 expression was significantly upregulated in human lung cancer tissues compared with normal lung tissue and was correlated with NSCLC progression. Moreover, Kaplan-Meier analysis 


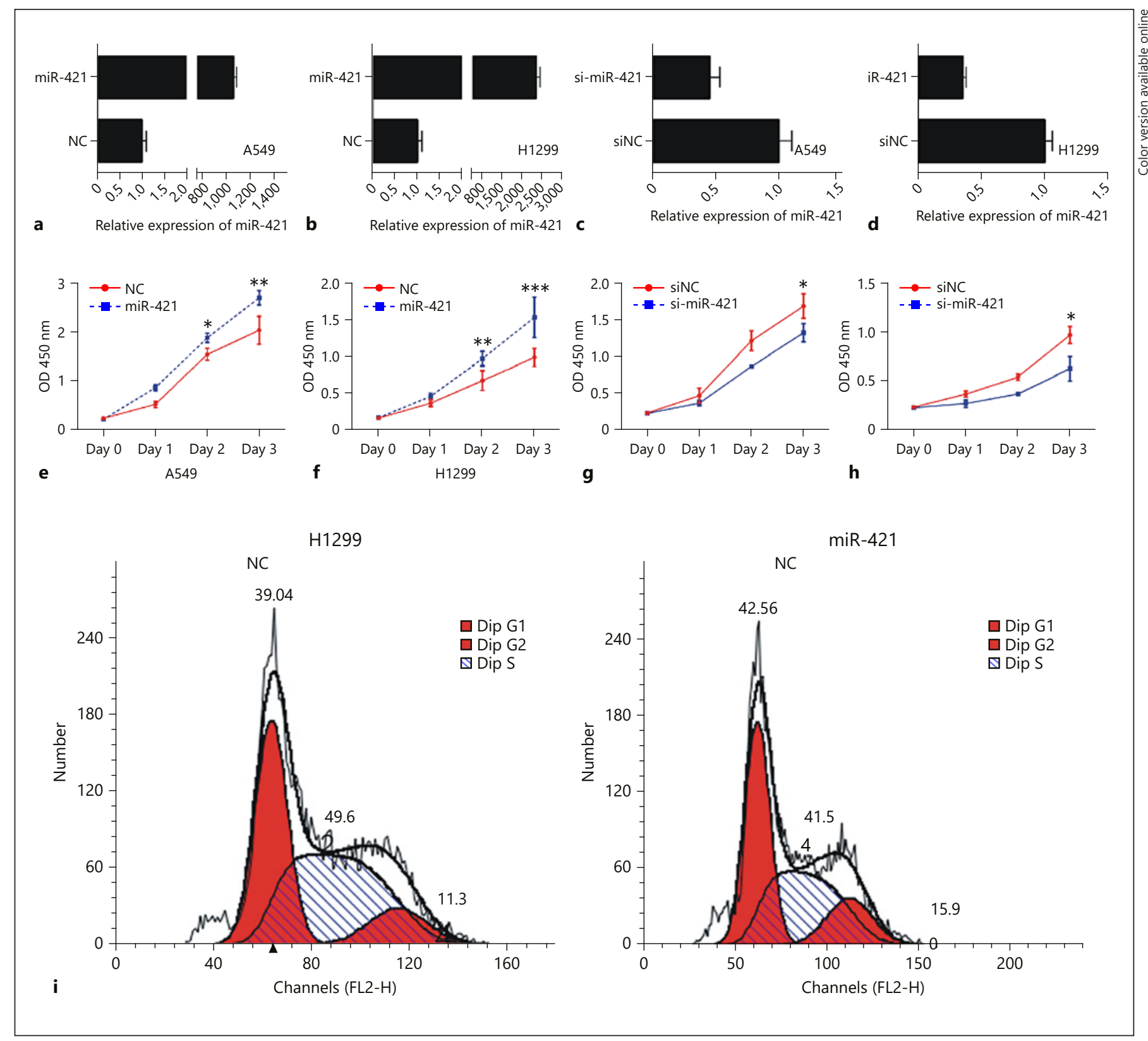

Fig. 5. miR-421 could promote NSCLC cell proliferation and cell cycle progression. a, b The transfection efficiency. Overexpression of miR-421 significantly promoted the proliferation of A549 (c) and H1299 cells (d). Overexpression of miR-421 significantly promoted the proliferation of A549 (e) and H1299 cells (f). Knock-

showed that higher miR-421 expression was significantly associated with shorter overall survival time in NSCLC.

Recent studies have revealed the important roles of miR-421 in human cancers and disease including breast

down of miR-421 significantly suppressed the proliferation of A549 (g) and H1299 cells (h). i Overexpression of miR-421-3p in H1299 significantly promoted cell cycle progression by decreasing the proportion of cells in G1 phase and increasing the proportion of cells in $\mathrm{S}$ phase. ${ }^{*} p<0.05,{ }^{* *} p<0.01,{ }^{* * *} p<0.001$.

cancer [21], gastric cancer [22], hepatocellular carcinoma [23], pancreatic cancer [24], LUAD [25], chronic kidney disease [26], adrenocortical tumors [27], osteosarcoma [13], nasopharyngeal carcinoma [28], cardiovascular disease [29], and inflammatory and thrombotic disorders 
Fig. 6. Overexpression of miR-421 suppressed insulin and MAPK signaling regulators. Overexpression of miR-421 significantly suppressed the expression of (a) RAPGEF2, (b) HK1, (c) RPS6KA3, and (d) TRAF6 in A549 and H1299 cells. By analyzing TCGA dataset, we found the miR421 expression was negatively correlated to the expression of (e) RAPGEF2, (f) HK1, (g) RPS6KA3, and (h) TRAF6 in NSCLC samples. ${ }^{*} p<0.05$.

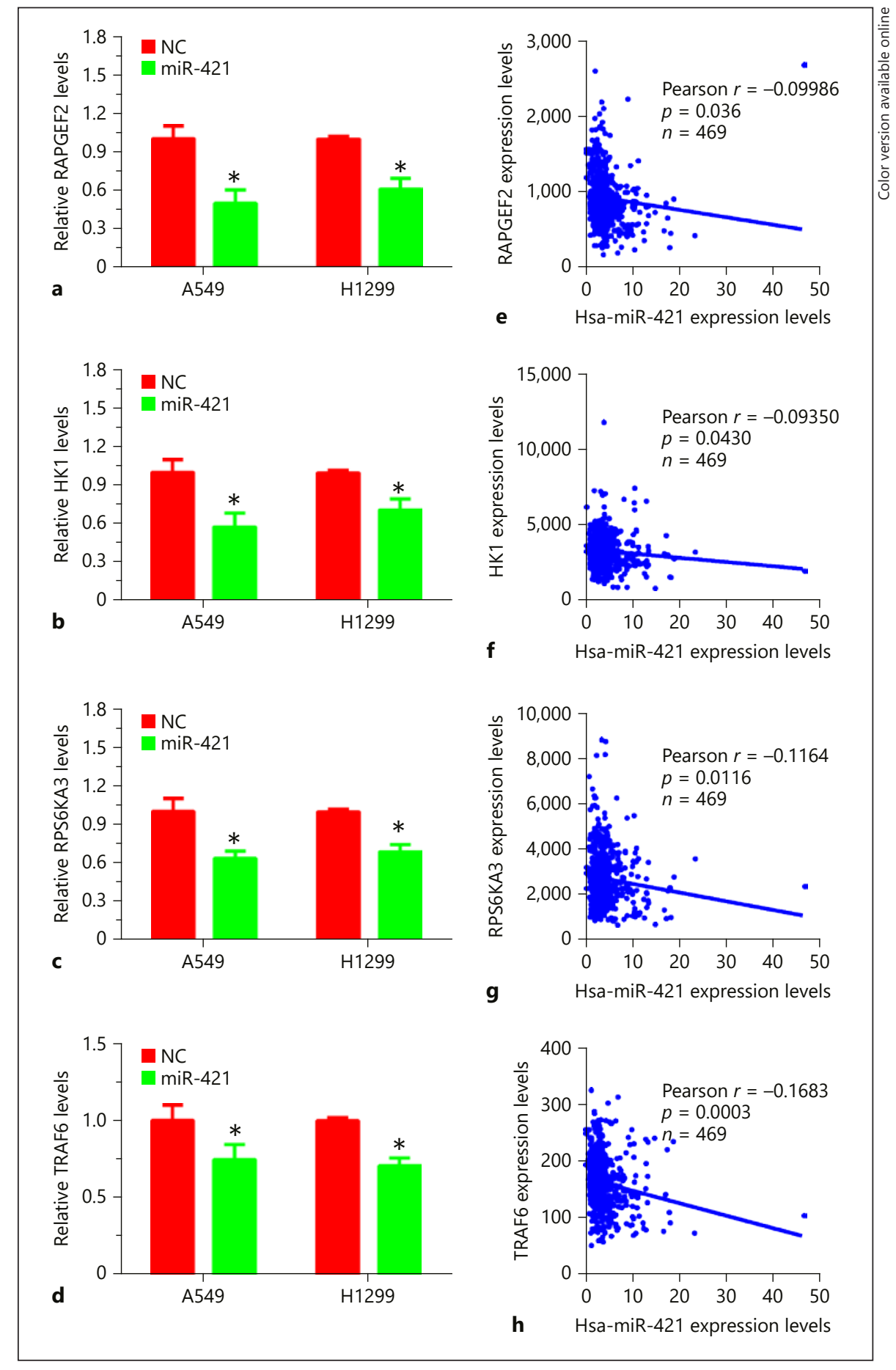

[30]. A recent study showed that miR-421 was upregulated in NSCLC samples and promoted cell proliferation. In this study, we performed gain of function assay and observed overexpression of miR-421 promoted NSCLC cell proliferation. Knockdown of miR-421 suppressed
NSCLC cell proliferation. We also performed flow cytometric analysis and found miR-421-3p could significantly promote $\mathrm{H} 1299$ cell cycle progression by decreasing the proportion of cells in G1 phase and increasing the proportion of cells in S phase. Previous studies showed 
miR-421 could regulate apoptosis, autophagy, proliferation, metastasis, epithelial - mesenchymal transition, metabolism, and radiotherapy resistance in various kinds of cancer by targeting different genes, such as TLR, caspase-10, caspase-3, MEF2D, PFKFB2, and FOXO4. However, the potential mechanism of miR-421 regulating NSCLC progression remained unknown. In the present study, we performed a bioinformatics analysis for miR421 to explore its potential mechanisms in NSCLC by using its targets. According to our analysis, we found that miR-421 was associated with transcription, protein monoubiquitination, positive regulation of G1/S transition of mitotic cell cycle, chromatin binding, poly(A) RNA binding, insulin signaling pathway, ubiquitin mediated proteolysis, and MAPK signaling pathway.

In order to explore the mechanisms of miR-421 underlying NSCLC progression, we detected the effect of miR421 on insulin and MAPK-signaling regulators, including HK1, RAPGEF2, TRAF6, and RPS6KA3. We found that overexpression of miR-421 significantly suppressed the expression of HK1, RAPGEF2, TRAF6, and RPS6KA3 in A549 and H1299 cells. HK1 was a type of hexokinases, which was observed to be overexpressed in colorectal cancer, melanoma, and gastric cancer. RAPGEF2 played a key role in the development and maintenance of epithelia. However, its roles in human cancers remained unclear. TRAF6 acted as an NF- $\kappa \mathrm{B}$ activator, whose expression was upregulated in urothelial bladder cancer, prostate cancer, and gastric cancer. TRAF6 was involved in regulating cancer cell metastasis and growth.

\section{Conclusion}

The present study showed that miR-421 was overexpressed in NSCLC and associated with a shorter overall survival time of NSCLC patients. Our results demonstrated that miR-421 might promote NSCLC cell proliferation and cell cycle progression. Taken together, the present study together with previous reports suggested that miR421 might be a promising prognostic and therapeutic target for NSCLC.

\section{Acknowledgment}

This work is supported by none.

\section{Statement of Ethics}

The authors have no ethical conflicts to disclose.

\section{Disclosure Statement}

The authors have no conflicts of interest to declare.

\section{Funding Sources}

This work is supported by none.

\section{References}

1 Siegel RL, Miller KD, Jemal A. Cancer Statistics, 2017. CA Cancer J Clin. 2017 Jan;67(1): 7-30.

2 Chen W, Zheng R, Baade PD, Zhang S, Zeng $\mathrm{H}$, Bray F, et al. Cancer statistics in China, 2015. CA Cancer J Clin. 2016 Mar-Apr;66(2): $115-32$.

3 Hou J, Aerts J, den Hamer B, van Ijcken W, den Bakker M, Riegman P, et al. Gene expression-based classification of non-small cell lung carcinomas and survival prediction. PLoS One. 2010 Apr;5(4):e10312.

4 Zhang WC, Chin TM, Yang H, Nga ME, Lunny DP, Lim EK, et al. Tumour-initiating cellspecific miR-1246 and miR-1290 expression converge to promote non-small cell lung cancer progression. Nat Commun. 2016 Jun;7(1): 11702.

5 Guan Y, Rao Z, Chen C. miR-30a suppresses lung cancer progression by targeting SIRT1. Oncotarget. 2017 Dec;9(4):4924-34.
6 Zhang JG, Wang JJ, Zhao F, Liu Q, Jiang K, Yang GH. MicroRNA-21 (miR-21) represses tumor suppressor PTEN and promotes growth and invasion in non-small cell lung cancer (NSCLC). Clin Chim Acta. 2010 Jun; 411(11-12):846-52.

7 Wen X, Han XR, Wang YJ, Wang S, Shen M, Zhang ZF, et al. MicroRNA-421 suppresses the apoptosis and autophagy of hippocampal neurons in epilepsy mice model by inhibition of the TLR/MYD88 pathway. J Cell Physiol. 2018 Sep;233(9):7022-34.

8 Hu TB, Chen HS, Cao MQ, Guo FD, Cheng XY, Han ZB, et al. MicroRNA-421 inhibits caspase-10 expression and promotes breast cancer progression. Neoplasma. 2018;65(1):49-54.

9 Wu JH, Yao YL, Gu T, Wang ZY, Pu XY, Sun $\mathrm{WW}$, et al. MiR-421 regulates apoptosis of BGC-823 gastric cancer cells by targeting caspase-3. Asian Pac J Cancer Prev. 2014;15(13): 5463-8.
10 Liu L, Cui S, Zhang R, Shi Y, Luo L. MiR-421 inhibits the malignant phenotype in glioma by directly targeting MEF2D. Am J Cancer Res. 2017 Apr;7(4):857-68.

11 Meng D, Yang S, Wan X, Zhang Y, Huang W, Zhao P, et al. A transcriptional target of androgen receptor, miR-421 regulates proliferation and metabolism of prostate cancer cells. Int J Biochem Cell Biol. 2016 Apr;73:30-40.

12 Li G, Song H, Chen L, Yang W, Nan K, Lu P. TUG1 promotes lens epithelial cell apoptosis by regulating miR-421/caspase- 3 axis in agerelated cataract. Exp Cell Res. 2017 Jul;356(1): 20-7.

13 Zhou S, Wang B, Hu J, Zhou Y, Jiang M, Wu $\mathrm{M}$, et al. miR-421 is a diagnostic and prognostic marker in patients with osteosarcoma. Tumour Biol. 2016 Jul;37(7):9001-7. 
14 Jiang Z, Guo J, Xiao B, Miao Y, Huang R, Li $\mathrm{D}$, et al. Increased expression of miR-421 in human gastric carcinoma and its clinical association. J Gastroenterol. 2010;45(1):17-23.

15 Livak KJ, Schmittgen TD. Analysis of relative gene expression data using real-time quantitative PCR and the 2(-Delta Delta C(T)) Method. Methods. 2001 Dec;25(4):402-8.

16 Huang W, Sherman BT, Lempicki RA. Bioinformatics enrichment tools: paths toward the comprehensive functional analysis of large gene lists. Nucleic Acids Res. 2009 Jan;37(1): $1-13$.

17 Agarwal V, Bell GW, Nam JW, Bartel DP. Predicting effective microRNA target sites in mammalian mRNAs. eLife. 2015 Aug;4:4.

18 Li JH, Liu S, Zhou H, Qu LH, Yang JH. starBase v2.0: decoding miRNA-ceRNA, miRNA-ncRNA and protein-RNA interaction networks from large-scale CLIP-Seq data. Nucleic Acids Res. 2014 Jan;42(Database issue):D92-7.

19 Yin H, Ma J, Chen L, Piao S, Zhang Y, Zhang $S$, et al. MiR-99a enhances the radiation sensitivity of Non-Small cell lung cancer by targeting mTOR. Cell Physiol Biochem. 2018; 46(2):471-81.
20 Huang RS, Zheng YL, Li C, Ding C, Xu C, Zhao J. MicroRNA-485-5p suppresses growth and metastasis in non-small cell lung cancer cells by targeting IGF2BP2. Life Sci. 2018 Apr; 199:104-11.

21 Wang Y, Liu Z, Shen J. MicroRNA-421-targeted PDCD4 regulates breast cancer cell proliferation. Int J Mol Med. 2019 Jan;43(1):26775.

22 Zhou H, Xiao B, Zhou F, Deng H, Zhang X, Lou Y, et al. MiR-421 is a functional marker of circulating tumor cells in gastric cancer patients. Biomarkers. 2012 Mar;17(2):104-10.

$23 \mathrm{Lu} \mathrm{M}$, Kong X, Wang H, Huang G, Ye C, He Z. A novel microRNAs expression signature for hepatocellular carcinoma diagnosis and prognosis. Oncotarget. 2017 Jan;8(5):877584.

24 Hao J, Zhang S, Zhou Y, Liu C, Hu X, Shao C. MicroRNA 421 suppresses DPC4/Smad4 in pancreatic cancer. Biochem Biophys Res Commun. 2011 Mar;406(4):552-7.

25 Cinegaglia NC, Andrade SC, Tokar T, Pinheiro M, Severino FE, Oliveira RA, et al. Integrative transcriptome analysis identifies deregulated microRNA-transcription factor networks in lung adenocarcinoma. Oncotarget. 2016 May; $7(20): 28920-34$
26 Trojanowicz B, Imdahl T, Ulrich C, et al. Circulating miR-421 targeting leucocytic angiotensin converting enzyme 2 is elevated in patients with chronic kidney disease. Nephron. 2019;141(1):61-74

27 Koperski Ł, Kotlarek M, Świerniak M, Kolanowska M, Kubiak A, Górnicka B, et al. Nextgeneration sequencing reveals microRNA markers of adrenocortical tumors malignancy. Oncotarget. 2017 Jul;8(30):49191-200.

28 Chen L, Tang Y, Wang J, Yan Z, Xu R. miR421 induces cell proliferation and apoptosis resistance in human nasopharyngeal carcinoma via downregulation of FOXO4. Biochem Biophys Res Commun. 2013 Jun;435(4):74550.

29 Lambert DW, Lambert LA, Clarke NE, Hooper NM, Porter KE, Turner AJ. Angiotensinconverting enzyme 2 is subject to post-transcriptional regulation by miR-421. Clin Sci (Lond). 2014 Aug;127(4):243-9.

30 Marchand A, Proust C, Morange PE, Lompré AM, Trégouët DA. miR-421 and miR-30c inhibit SERPINE 1 gene expression in human endothelial cells. PLoS One. 2012;7(8):e44532. 